Original Article - Clinical Science

\title{
Materials in the vitreous during cataract surgery: nature and incidence, with two cases of histological confirmation
}

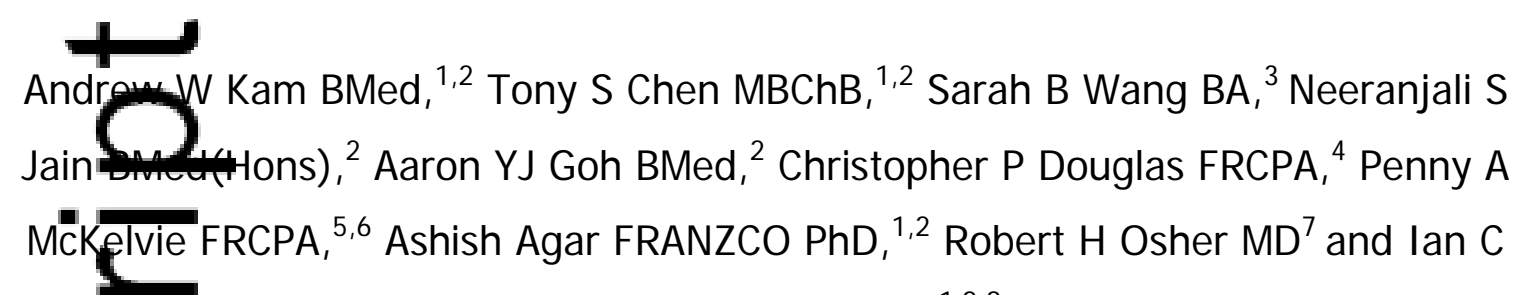

\section{U}

Francis FRANZCO PhD ${ }^{1,2,8}$

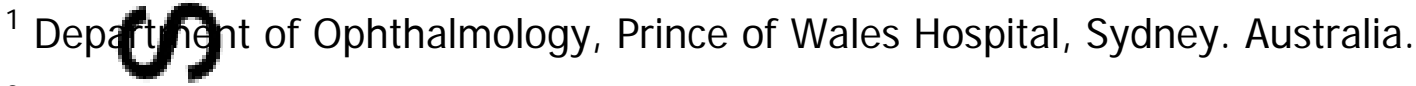

2 The University of New South Wales, Sydney. Australia.

${ }^{3}$ The Unversity of Sydney, Sydney. Australia.

${ }^{4}$ Histc path Specialist Pathologists, Sydney. Australia.

${ }^{5}$ The University of Melbourne, Melbourne. Australia.

${ }^{6}$ St Vildad's Hospital, Melbourne. Australia.

${ }^{7}$ Cincrati Eye Institute, Cincinnati. OH, USA.

${ }^{8}$ Opdimic Surgery Centre, Sydney. Australia.

Corres andence: Associate Professor Ian C. Francis, Chatswood Eye Specialists

Suite Legel 2, 38b Albert Ave, Chatswood, NSW, 2067, Australia

Email: iancfrancis@gmail.com

Shorturnmg title: Materials in the vitreous

Received 8 April 2016; accepted 7 J une 2016

Conflict of interest: None

Funaing bources: None

This is the author manuscript accepted for publication and has undergone full peer review but has not been through the copyediting, typesetting, pagination and proofreading process, which may lead to differences between this version and the Version of Record. Please cite this article as doi: 10.1111/ceo.12791

This article is protected by copyright. All rights reserved. 


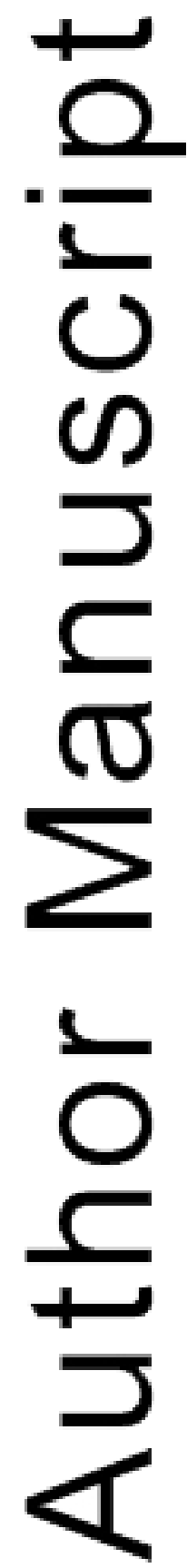

This article is protected by copyright. All rights reserved. 


\section{ABSTRACT}

Background: To identify and classify materials in the vitreous (MIV) observed during phacoemulsification cataract surgery (phaco).

Design: Prospective, consecutive, observational case series at one ophthalmic day surgèty In Sydney, Australia

Partidiparts: 767 consecutive phaco cases. Cases were excluded if there was poster verule rupture or vitreous loss intraoperatively.

formor each patient, age, gender, baseline corrected distance visual acuity (CDVA , prosence of pseudoexfoliation, nuclear sclerosis grade and phacoemplafification ultrasound time were recorded. The relationship between these variables and MIV was evaluated with regression analysis. Two patients with MIV developed an Acute Intraoperative Rock-hard Eye Syndrome. In these two patients, pars parneedle aspiration of retrolenticular fluid was performed to re-establish normantmutracular pressure. Histology was undertaken to compare this fluid with knowr 1 material retrieved from the Fluid Management System bags in two unreladeases.

Main owtome measurements: Presence of MIV during phaco.

Results: MIV were observed in either Berger's space or the anterior vitreous (AV) in 386 eyes ( $50.3 \%$ of cases); the majority was putatively lens material $(46.5 \%$ of all cases) Digment and OVD were seen in the AV in $9.8 \%$ and $1.7 \%$ of cases, respecty. Logistic regression analysis demonstrated that higher nuclear sclerosis grade $p=0.025)$, male gender $(p=0.003)$, and greater age $(p=0.016)$ were predictive of the presence of MIV. Histological assessment with light microscopy and birefrime techniques identified the MIV as lens material.

Conclusion: MIV were seen in $50.3 \%$ of phaco cases. It has been histologically demerisi ated that lens materials can be introduced into the AV during phaco. 
Keywords: Lens, cataract surgery, cataract

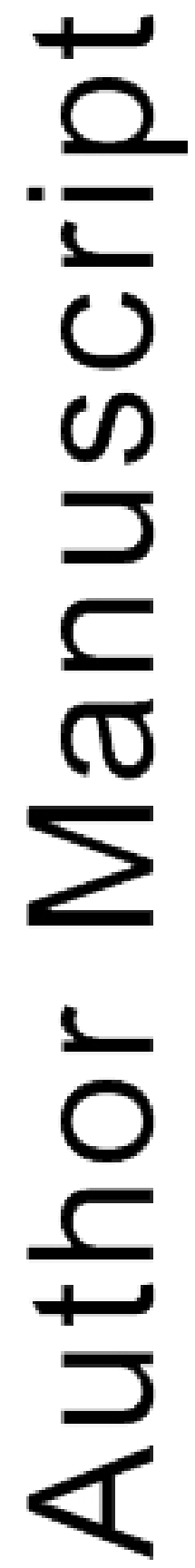

This article is protected by copyright. All rights reserved. 


\section{NTRODUCTI ON}

Modern phacoemulsification cataract surgery (phaco) almost always produces excellent surgical and visual outcomes. Despite an intact posterior capsule, materials foreign to the anterior vitreous (AV) are not infrequently visualised in the AV or Bergetrs space during phaco. ${ }^{1-3}$

\section{은}

Based appearance, previous reports have postulated that these materials are comprised primarily of lens fragments. ${ }^{1,3}$ The only histological investigation of this m fteril in the literature, however, undertaken in 30 patients undergoing combipes phaco and vitrectomy surgery, was unable to obtain histological evidence of lens material in the $\mathrm{AV}^{2}$

$\longrightarrow$

The abeene of definitive histological evidence has led to ongoing debate regarding the possiomity of lenticular fragment access into the vitreous cavity, 2,4,5 and indeed over v (htt)er materials can enter the vitreous at all. ${ }^{2}$ The authors of the previous histchinvestigation postulated that these commonly observed 'materials' might only be inithickers of lens material, such as titanium fragments shed from the phaco tip due to wear and tear, or AV condensations. ${ }^{2}$

These paterials, termed "Materials In the Vitreous" (MIV) have been commonly obsen our group. In addition to putative lens material, our group has observed putati e pigment, ophthalmic viscoelastic device (OVD), and air in the AV. This study was undertaken to determine the incidence, and classify the nature of, MIV obsend the AV during uncomplicated phaco. Further, the study reports two cases ominstological examination of MIV and, to our knowledge, the first histological confina ion of lenticular fragments in the AV during uneventful phaco. 


\section{METHODS}

This study was a prospective, consecutive, single-surgeon cohort study of 767 eyes undergoing phaco at an ophthalmic day surgery centre in Sydney, Australia between J anuary 2010 and December 2012. Cases were excluded if there was a posterior capsule rupture or vitreous loss intraoperatively. Ethics approval was granted by the Unive fity New South Wales, Australia (Approval: HC13220). The procedures and documetion were performed according to the tenets of the Declaration of Helsinki.

\section{fication and classification of MIV}

All patien underwent preoperative assessment according to the operating surgeon's hormal protocol, including slit lamp examination and intraocular pressure measurem nt. Evidence of vitreous condensations, vitreous syneretic cavities, posteritreous detachment and pigment in the $\mathrm{AV}$ were sought as part of the preoperative workup. If pigment were recognised preoperatively, careful inspection of the UDis and posterior segment for any source of the pigment, such as a retinal tear vinence of previous trauma, pseudoexfoliation (PXF) and pigment dispersion syndrome was carried out.

The following surgical procedure was employed in all cases: Two clear corneal incisionamere made at approximately $80^{\circ}$ from each other, aiming to optimise ergonomid comfort for the surgeon. The mainport incision was $2.2-2.4 \mathrm{~mm}$, and the sidepd $1.5 \mathrm{~mm}$. A 5.5mm capsulorrhexis, confirmed by the surgical assistant with calipers. was performed under dispersive OVD. In all procedures, the Infiniti Vision Systen ${ }^{2}$, Fort Worth, Texas) was utilised. Following nucleus hydrodissection and rotation, the nucleus was removed utilising the phaco stop and chop technique. ${ }^{6}$ Durie g ladrant removal, the aspiration flow rate was fixed at $30 \mathrm{cc} / \mathrm{min}$. Residual cortical fibres were loosened using the "Hydropolish" technique. ${ }^{7}$ Cortical removal 
was performed using automated irrigation/aspiration (I/A), followed by manual irrigation of residual cortical fibres on the posterior capsule. Posterior capsular polishing was used when necessary. A hydrophobic, one-piece acrylic intraocular lens (IOL) was implanted using a D-cartridge (Alcon ${ }^{\circledR}$ ) by means of a Monarch III IOL Injector (Alcon $®)$.

$+$

Once brtex had been removed to the surgeon's satisfaction, the surgeon and surgicastant collaboratively evaluated the presence, number and nature of MIV in eith Berger's space and/or the AV. This was achieved by direct observation throud th cataract surgical operating microscope.

C

All cases were classified into four putative classes of MIV (lens material, pigment, OVD, and ir) based on the appearance of each one (Figure 1). Each had been previomserved by the authors. The presence of each class of MIV, and the phacoemursification ultrasound time, were recorded. All data were documented immediafl by the OR team on a standard proforma in each case. ${ }^{8}$

Further detta collected included age, gender, ocular comorbidities, preoperative corrected distance visual acuity (CDVA) and cataract grade as per the Lens Opacities Classification System III (LOCS III). ${ }^{9}$

In the 0 operative period, and in the absence of relevant allergies, patients were given ffloxacin, fluorinated steroids and nonsteroidal anti-inflammatory drugs (NSAIPs) topically. The ofloxacin and NSAIDs were ceased at two weeks, and the fluorineted-steroids tapered over several weeks.

All prer ts were evaluated postoperatively for CDVA at one day, one week and one month following phaco. Slit lamp examination and intraocular pressure (IOP) 
measurement were carried out at each visit. Postoperative visual acuity assessment was performed with objective laser interferometric refraction. Manual retinoscopy and subjective refraction were performed if necessary. A thorough postoperative ophthalmological examination was repeated in cases where postoperative CDVA did not meet clinical expectations, or if the CDVA deteriorated between the first and subsequent postoperative visits. This examination included detailed inspection of the ocular surf.ce, visual fields to confrontation, pupillary assessment (to exclude a relativeffent pupillary defect), fundus examination and optical coherence tomograpiny (OCT).<smiles>O</smiles>

\section{Histolpgigal investigation of MIV}

Two cases of the 767 with MIV experienced Acute Intraoperative Rock-hard Eye Syndrome AI RES) during phaco. ${ }^{10}$ In both cases of AIRES, MIV were visualised. Both ceerof AIRES were successfully managed with emergent pars plana needle aspiratron of retrolenticular fluid with a 23 -gauge needle and a $3 \mathrm{~mL}$ syringe. ${ }^{10}$ Seren iptously, this facilitated the collection of MIV for histological examination. Samasthe MIV were sent to two independent, consultant histopathologists (CPD and AM). Both samples underwent haematoxylin and eosin staining, and were assessed using birefringence techniques.

\section{1}

The MIVspecimens collected from the AI RES cases were compared with known lens materidrieved from the Fluid Management System (Alcon ${ }^{\circledR}$ ) bags from two unrela ed cases following phaco.

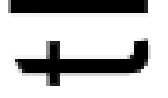

\section{Statistien analysis}

Binary Togrstic regression analysis was performed to identify any relationship betven presence of MIV and age, gender, baseline CDVA, presence of PXF, nuclear 
sclerosis grade $(\text { LOCS III })^{9}$ and phaco time. All statistical analyses were performed using SPSS ${ }^{\circledR}$ (version 22; SPSS Inc., Chicago, III).

\section{RESULTS}

The study cohort comprised 767 prospective, consecutive phaco surgeries. All cases were icluded as posterior capsular rupture or vitreous loss did not occur in any of the eorean patient age was 70.9 years (SD: 9.1 years) and $61.8 \%$ of the cohortwersfemale (474 cases).

U

Some form of MIV was seen in 386 cases (50.3\%). Putative lens material was most commonly observed, being visualised in 357 cases (46.5\%) (Figure 1a, b). Pigment MIV was sfen in 75 cases (9.8\%) (Figure 1c), while OVD MIV was observed in 13 cases (Figure $1 d, e)$. Though the authors have previously seen, and occasionarity continue to see, air in the AV, none was seen in the study cohort (Figur (f) These findings are summarised in Table 1. It should be emphasised that differyes of MIV can be observed concurrently (Figure 1 c, d). In our cohort, cortex ant pigment MIV were seen concurrently in 60 cases (7.8\%); cortex and OVD in 8 cases (1.0\%); and cortex, pigment and OVD MIV in 5 cases (0.7\%).

\section{1}

Binary pesistic regression analysis demonstrated that higher nuclear sclerosis grade ( $p=0.055$, male gender $(p=0.003)$, and greater age $(p=0.016)$ were predictive of the pr sence of any MIV. Higher nuclear sclerosis grade $(p=0.006)$ and male gender ( $p=0.010)$ predicted the presence of lens material in the AV. Presence of PXF alone predictigment in the AV ( $p=0.007)$. Higher nuclear sclerosis grade alone predictea UVD in the AV ( $p=0.019)$. Phacoemulsification ultrasound time and baseine CDVA had no significant predictive value for any form of MIV. 


\section{Case with cystoid macular oedema}

One patient developed clinically significant cystoid macular oedema (CMO). This was diagnosed by a deterioration in CDVA from 6/4 (confirmed at the one day and one week followup) to 6/18 at one month. Fundus examination and OCT confirmed the diagnosis. Treatment led to resolution of this patient's CMO, and CDVA recovered to $6 / 4$ at'

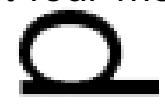

\section{Histologieal examination of MIV}

Retrolefticelar fluid samples containing MIV from each of the two AIRES ${ }^{10}$ patients were ristolygically compared with known lens material derived from the Fluid Managengent System bags of two unrelated cases. Haematoxylin and eosin staining, and examination under polarized light, demonstrated the MIV to display characteris ics identical to the known lens material derived from the bags. It was subsequencluded that both retrolenticular fluid samples contained lens materiar(Trgures $2 \mathrm{a}, \mathrm{b}, \mathrm{c}, \mathrm{d}$ ).

\section{ర}
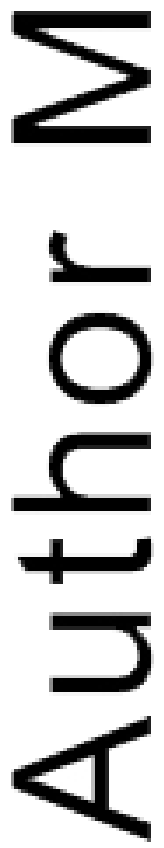


\section{DISCUSSION}

To our knowledge, this study is the first to provide histological confirmation of the presence of lens material in the AV during routine phaco. Further, this study has identified older age, male gender and more advanced nuclear sclerosis grade as risk factors for MIV. Although MIV has been observed by many cataract surgeons, the high ffequency of MIV reported (50.3\%) is of a higher rate than the authors believe many sungeons would intuitively estimate.

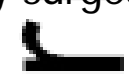

Phaco of dunser nuclei, requiring higher energy output, may contribute to lens fragmentfy moving more vigorously in the anterior chamber. In addition, it is entirely possible that one of the techniques for freeing tenacious cortex, direct irrigation with a straight $d$ a curved cannula of the inferior or superior capsular bag fornix, ${ }^{7}$ may

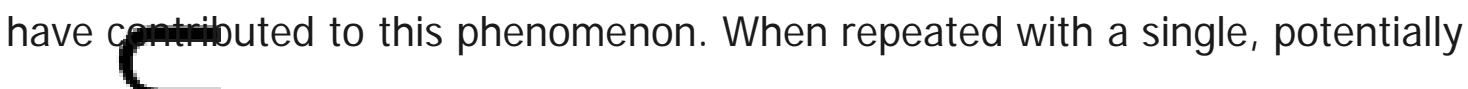
poweruruget of balanced salt irrigated through a syringe and a cannula, there may well h 1 been transgression of the zonular apparatus by the balanced salt solution and malleces of lens material.

As mentioned in the Introduction, it was suggested previously by Liu et al. that materials observed in the vitreous during cataract surgery may be titanium fragments shed from the phaco tip due to wear and tear, ${ }^{2}$ or AV condensations, which, nobserved through an operating microscope, may possibly resemble lens mater a ${ }^{2}$ The authors can confidently say that at no stage before, during or after this studv. have the surgeons in our group seen 'metal' in the AV, nor AV condeneations, that could have been confused with the four types of MIV documented by our group.

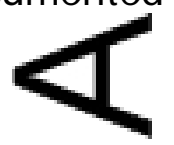


This study found a high rate of MIV in normal eyes with intact posterior capsules and zonules. It therefore suggests the existence of an anatomical conduit allowing movement of fluid and particulate matter between the anterior chamber and posterior segment in some normal eyes. Indeed, such a conduit has been previously suggested by cases of unexpected staining of the posterior surface of the posterior capsule by trypan blue during phaco in normal eyes. ${ }^{11}$ However, to our knowledge, this has ye to be formally identified anatomically.

It is postrated that the conduit by which MIV enter the vitreous cavity is through the intersti)es between the intact zonular fibres during nuclear phacoemulsification and cotipg I/A. Such a conduit would also account for the retrolenticular misdirection of fluid associated with the phenomenon of AIRES. ${ }^{10}$ The authors emphasise that such a conduit exists in the absence of zonular deficiencies, as no cases cohort were noted to have intraoperative zonular instability.

The ri 2 tors for MIV identified in this study were higher nuclear sclerosis grade, mal and ane, and in the case of pigment MIV, the presence of PXF. These risk factors 2 also associated with zonular dehiscence and weakness. ${ }^{12,13}$ This suggests that in patients with these risk factors, the zonular fibres may be physically or biochemically altered. This occurs despite them appearing normal under the operatipg-picroscope, and demonstrating no axial shift of the capsulorrhexis margin. This zowuar fibre alteration may allow anterior segment materials to bypass the zonula fibres more readily, and enter the posterior segment, resulting in MIV. Supporting this notion are previous case reports of inadvertent staining of the vitreous trypan blue during phaco. This has been observed in eyes where previous ocular trauma or pre-existing PXF have caused zonular apparatus weak 14 .17 
Further, a report out of Manchester, England, documented a case in which anterior capsular irrigation with trypan blue led to blue discolouration of the posterior surface of the posterior capsule in a previously vitrectomised patient. ${ }^{18}$ Indeed, the authors' vitreoretinal colleagues report this as a not infrequent occurrence. In the current study cohort, one patient who had previously undergone vitrectomy likewise deveioped blue discolouration of the posterior surface of the posterior capsule follow anterior capsular irrigation with trypan blue. Interestingly, in this case, following balanced salt irrigation continued over three more minutes, there was near omplete resolution of the blue posterior capsule discolouration. The surger wa then completed uneventfully. This represents further indirect evidence of the aromic conduit between the anterior chamber and the vitreous cavity.

CMO is red gnised as one of the leading causes of poor visual outcomes after uncompleated phaco, and may result in permanent visual loss. ${ }^{19}$ The pathogenesis of CMG IS Considered most likely to be inflammatory. ${ }^{20-23}$ Inflammation may result in disrup(iof $f$ the blood-aqueous barrier with a subsequent increase in iris vascular leakend disruption of the blood-retinal barrier. ${ }^{20-23}$ The posterior segment of the eye is nomally not exposed to lens antigens, as is the case with the other intraocular structures. As MIV are foreign to the posterior segment, they therefore represent a potential new immunological stimulus, to which the posterior segment is naïve. Thay may therefore contribute to intraocular inflammation following cataract surger reover, the ingress of macroscopic MIV suggests that other, microscopic mater as released during phacoemulsification, such as free radicals or lens epithelial cells mav also be introduced into the vitreous. ${ }^{24-27}$ These materials may possibly play a postoperative complications.

The case of CMO encountered in our study was not associated with MIV. Due to the small size and low rate of clinically significant CMO ( 1 of $767,0.13 \%)$ in the 
study cohort, however, our study was not adequately powered to examine the relationship between MIV and CMO. As such, the authors are unable to draw conclusions as to the significance of MIV in relation to postoperative complications.

Thus, the presence of MIV demonstrates the existence of a conduit between the anterior and posterior segments of the eye, and may be associated with physically altered or iochemically altered zonular fibres. Therefore, in cases where MIV are obsernalit may be helpful for surgeons to exercise increased care when completing the pherespecially when irrigating with balanced salt solution. MIV may also sugge $t$ th need for increased vigilance in postoperative management, and particyary for surgery on the second eye.

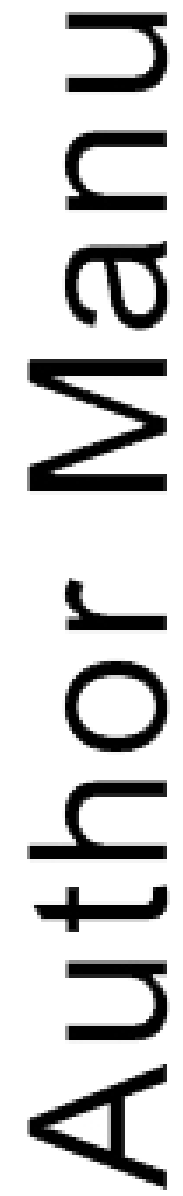


In conclusion, this study has demonstrated that MIV may be observed in as many as $50.3 \%$ of uncomplicated phaco cases. MIV consists mainly of lens material, but pigment and OVD were also recognised. Although the authors have previously and subsequently observed air in the AV, no air was visualised in this study cohort. Age, male gender and higher nuclear sclerosis grade were predictive of MIV. The finding of PXIWas predictive of pigment MIV. Confirmation that the histological nature of MIV S A material was obtained in two cases. Further studies may elucidate of the sigmificence of MIV in operative and postoperative phaco complications.

1

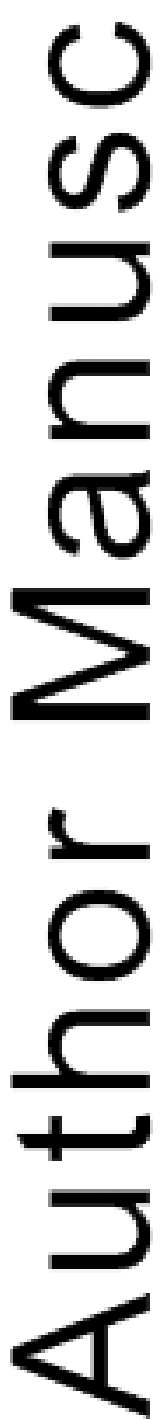

This article is protected by copyright. All rights reserved. 


\section{REFERENCES}

1. Ang A, Menezo i Rallo V, Shepstone L, et al. Retrocapsular lens fragments after uneventful phacoemulsification cataract surgery. J Cataract Refract Surg 2004; 30: 849-53.

2. Liư LI, Lee VY, Li CL, et al. Retrocapsular lens matter in uneventful phacomulification: does it really exist? Clin Experiment Ophthalmol 2008; 36: 31-5.

3. Novetrolenticular lens fragments--a problem of phacoemulsification?]. Cesk Sivoftalmol 1999; 55: 268-73.

4. Liu CT, Thoi PC, Chan WM, et al. Retrocapsular lens fragments after uneventful phacoem

5. Oh YH, Chuck RS, Do J R, et al. Vitreous Hyper-Reflective Dots in Optical Coherence Tomography and Cystoid Macular Edema after Uneventful Phacomification Surgery. PLoS One 2014; 9: e95066.

6. KoctrTs, Katzen LE. Stop and chop phacoemulsification. J Cataract Refract Surg 1994; 2066-70.

7. Vhan XM, Amjadi S, et al. Hydropolish: a controlled trial on a technique to eradiente residual cortical lens fibers in phacoemulsification cataract surgery. Eur J Ophthalmol; 25: 571-4.

8. Ng QT, Rowe NA, Francis IC, et al. Intraoperative complications of 1000 phacoenaysification procedures: a prospective study. J Cataract Refract Surg 1998; 24: 1350.

9. Chy ack L, Jr, Wolfe JK, Singer DM, et al. The Lens Opacities Classification System III. The Longitudinal Study of Cataract Study Group. Arch Ophthalmol 1993; 111: 831-6.

10. Lauro, Montfort J M, Sim BW, et al. Acute intraoperative rock-hard eye synd and its management. J Cataract Refract Surg 2014; 40: 799-804. 
11. Burkholder BM, Srikumaran D, Nanji A, et al. Inadvertent trypan blue posterior capsule staining during cataract surgery. Am J Ophthalmol 2013; 155: 625-28.

12. Bornfeld N, Spitznas M, Breipohl W, et al. Scanning electron microscopy of the zonule of Zinn. Graefes Arch Clin Exp Ophthalmol 1974; 192: 117-29.

13. Yuen CYF, Rao SK, Li EYM, et al. Capsular tension ring for zonular dehiscence in comblined phacoemulsification and pars plana vitrectomy with or without gas tamporade. HKJ Ophthalmol 2014; 15: 35-37.

14. Chering PK, Raj SM, Vasavada AR. Inadvertent Staining of the Vitreous with Tryparfore J Cataract Refract Surg 2004; 30: 274-76.

15. Gd (ur A) Kayarkar W. I nadvertent vitreous staining. J Cataract Refract Surg 2005;

31: 648

16. Kheirkhah A, Nazari R, Roohipour R. Inadvertent vitreous staining with trypan blue in pse Idoexfoliation syndrome. Arch Ophthalmol 2010; 128: 1372-73.

17. All Russell SR, Schluter ML, et al. Retained posterior segment indocyanine green wye after phacoemulsification. J Cataract Refract Surg 2006; 32: 357-60. 18. Bi Cf allWW, Raynor MK, Turner GS. Inadvertent staining of the posterior lens caps trypan blue dye during phacoemulsification. Arch Ophthalmol 2001;

\section{9: 10\%-83.}

19. Mentes J, Erakgun T, Afrashi F, et al. Incidence of cystoid macular edema after uncomplicated phacoemulsification. Ophthalmologica 2003; 217: 408-12.

20. Miyaln K, Masuda K, Shirato S, et al. Comparison of diclofenac and fluorormolone in preventing cystoid macular edema after small incision cataract surger : a multicentered prospective trial. Jpn J Ophthalmol 2000; 44: 58-67. 21. Almeid DR, J ohnson D, Hollands $H$, et al. Effect of prophylactic nonsteroidal antiinflematory drugs on cystoid macular edema assessed using optical coherence tomograpriy quantification of total macular volume after cataract surgery. J Cataract Refresurg 2008; 34: 64-9. 
22. Asano S, Miyake K, Ota I, et al. Reducing angiographic cystoid macular edema and blood-aqueous barrier disruption after small-incision phacoemulsification and foldable intraocular lens implantation: multicenter prospective randomized comparison of topical diclofenac $0.1 \%$ and betamethasone $0.1 \%$. J Cataract Refract Surg 2008; 34: 57-63.

23. Mátnys KC, Cohen KL. Impact of nepafenac $0.1 \%$ on macular thickness and postoperat ve visual acuity after cataract surgery in patients at low risk for cystoid macellema. Eye (Lond) 2010; 24: 90-6.

24. Ganturer J M, Aust SD. Quantification of hydroxyl radical produced during phaco mulification. J Cataract Refract Surg 2009; 35: 2149-53.

25. Holst Rolfsen W, Svensson B, et al. Formation of free radicals during phacoemuísification. Curr Eye Res 1993; 12: 359-65.

26. Camerd $\mathrm{n}$ MD, Poyer JF, Aust SD. I dentification of free radicals produced during phacoemification. J Cataract Refract Surg 2001; 27: 463-70.

27. Vasavada A, Johar K, Praveen $M$, et al. Confirmation of the presence of lens epithe (a) Ills in the anterior chamber after phacoemulsification. Eye (Lond) 2009;

\section{3:}
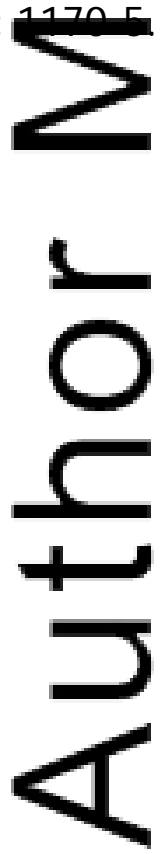


\section{FIGURE LEGENDS}

\section{Figure 1:}

a. Single piece of lens material (arrow) in the anterior vitreous during phacoemulsification cataract surgery.

b. Mütriple pieces of lens material noted in the anterior vitreous towards completion of irrigtion/aspiration in a grade 4 nuclear sclerotic cataract. Note residual subincievenal cortex is still to be removed.

c. Pigntering (thick arrows) along with lens material (thin arrow) in the anterior vitreo(s.

d. Leng naterial (thick arrow) and OVD (thin arrow) in the anterior vitreous.

e. Lens material (thick arrow) and OVD (thin arrow) in the anterior vitreous. Note that in pand e, the lens material and the OVD have moved relative to each other within vitreous (compared with d).

f. Six discrete air bubbles seen in the anterior vitreous during uneventful phacoemulsification cataract surgery. Their location is confirmed as this image was taken immediately prior to inflation of the anterior chamber with balanced salt solution at the completion of surgery. Posterior capsular folds are seen anterior to the air bubbles in the anterior vitreous. Air was not observed in the study cohort. This photograph was taken during surgery on a patient with a LOCS III grade 2+ cataract in September 2015. This patient's corrected distance visual acuity was 6/4 at one day, one week and one month postoperatively.

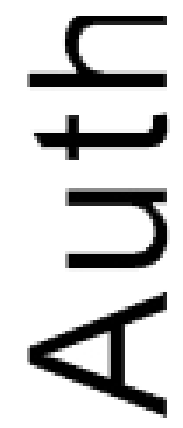




\section{Figure 2:}

a, b. Light microscopy (haematoxylin and eosin [x100]) showing the histology of the material in the vitreous obtained from retrolenticular fluid, consistent with known lens material.

c, d. Haematoxylin and eosin (x100) with polarised light showing the material in the vitreots. Both of these specimens demonstrated the same haematoxylin and eosin stainin and birefringence as lens material obtained from the Fluid Management Systentorgs.

1
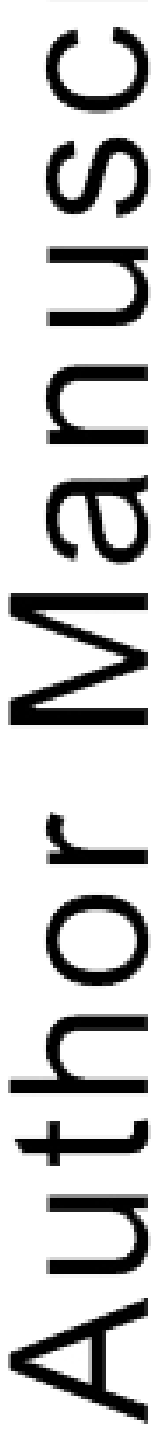


\section{TABLE}

Table 1: Classification and incidence of materials in the vitreous (MIV).

\begin{tabular}{|lrr|}
\hline Type of MIV & Frequency (n) & Incidence (\% ) \\
\hline Lens rtaterial & 357 & $46.5 \%$ \\
\hline Pigme & 75 & $9.8 \%$ \\
\hline OVD & 13 & $1.7 \%$ \\
\hline Air & 0 & $0.0 \%$ \\
\hline
\end{tabular}

OVD $=$ gphthalmic Viscoelastic Device.

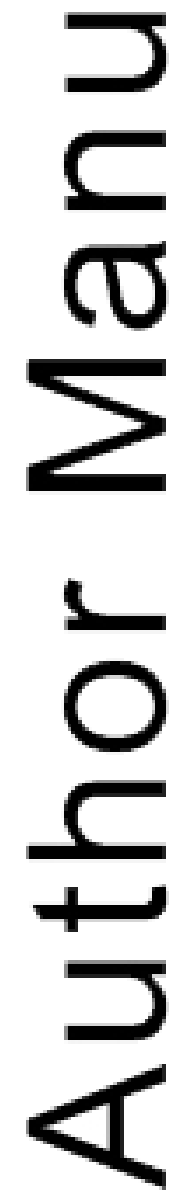




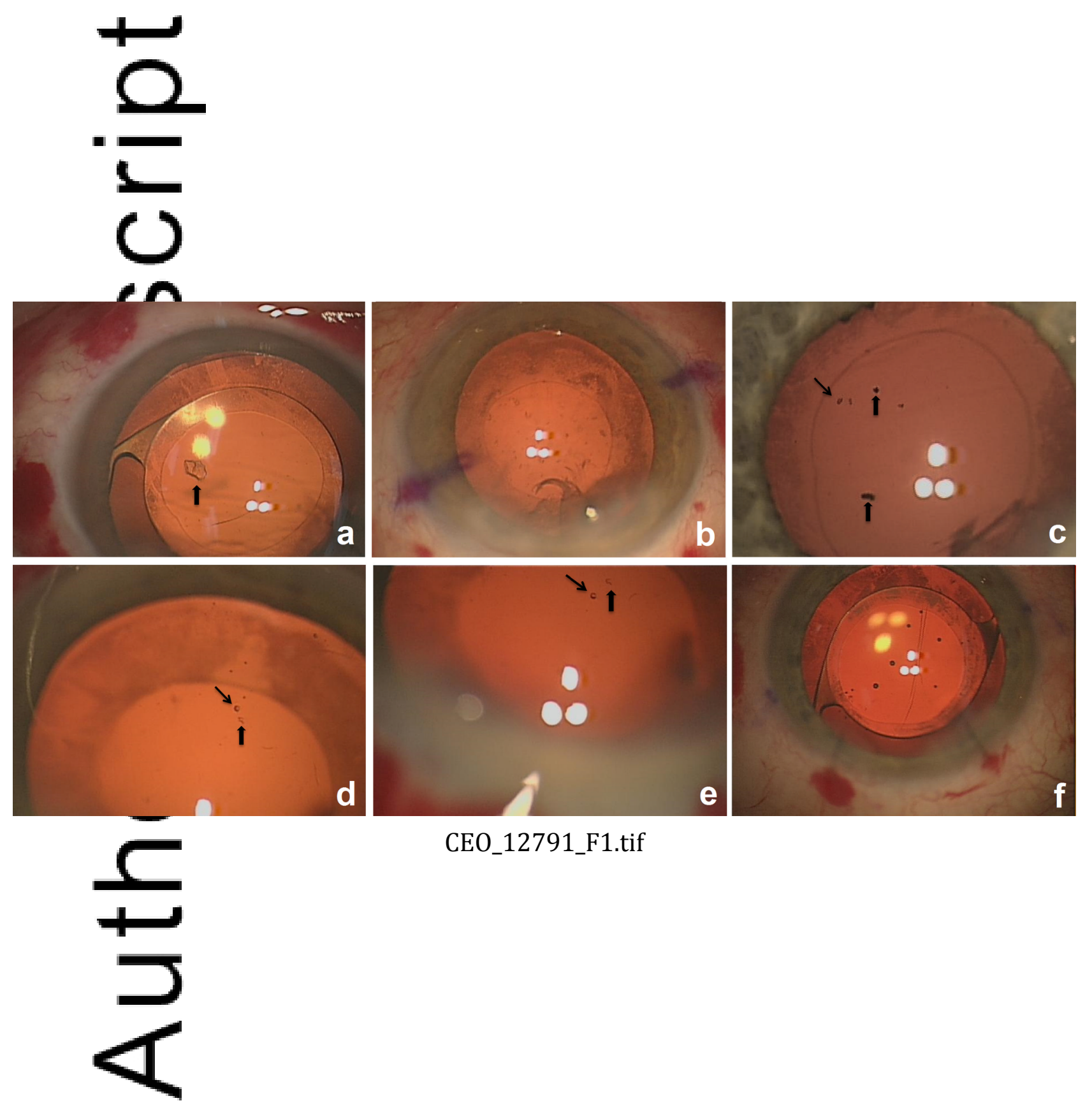

This article is protected by copyright. All rights reserved. 


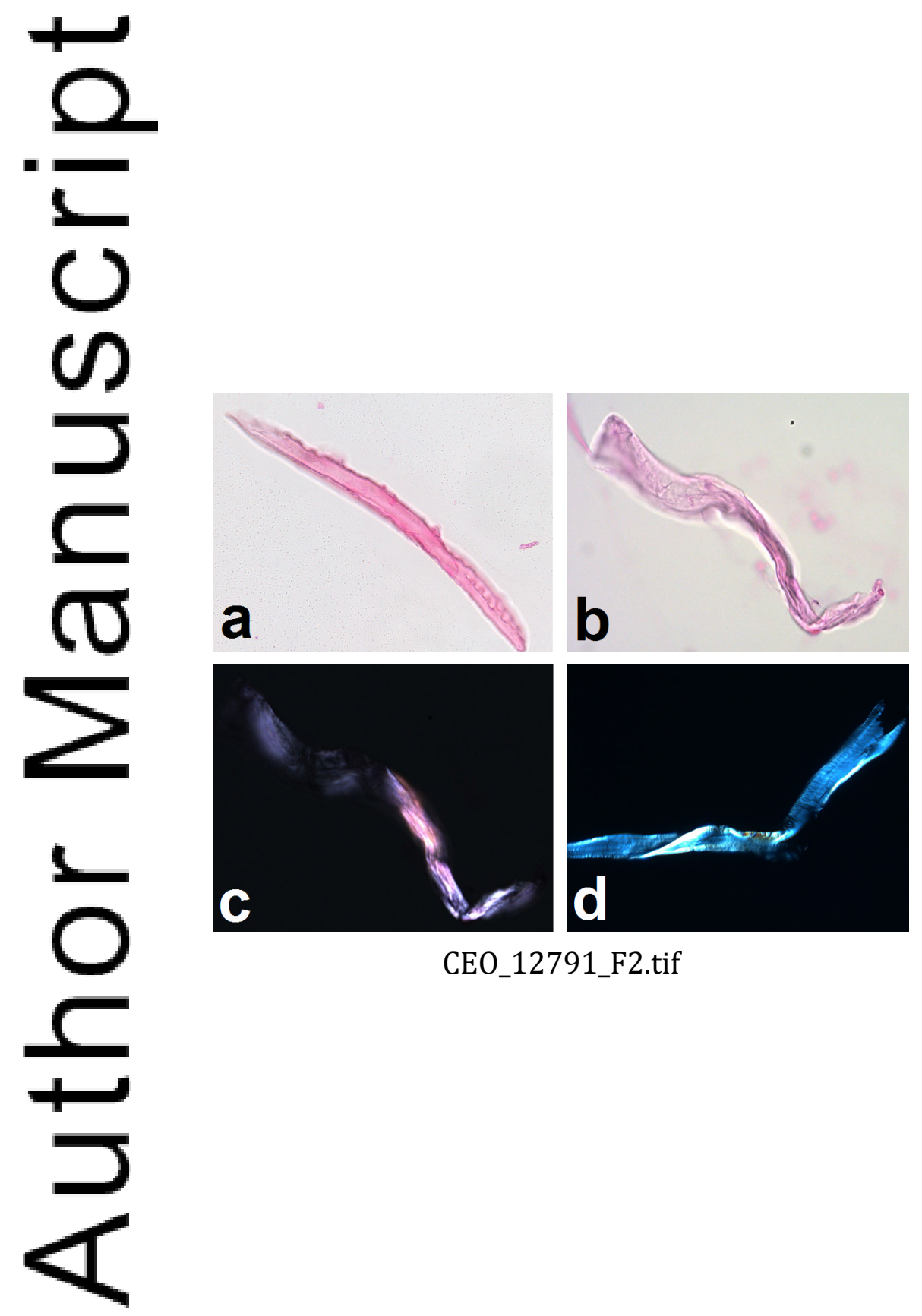

This article is protected by copyright. All rights reserved. 


\section{University Library}

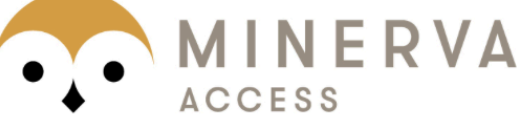

A gateway to Melbourne's research publications

Minerva Access is the Institutional Repository of The University of Melbourne

Author/s:

Kam, AW;Chen, TS;Wang, SB;Jain, NS;Goh, AYJ;Douglas, CP;McKelvie, PA;Agar, A;Osher, $\mathrm{RH}$;Francis, IC

Title:

Materials in the vitreous during cataract surgery: nature and incidence, with two cases of histological confirmation

Date:

2016-12-01

Citation:

Kam, A. W., Chen, T. S., Wang, S. B., Jain, N. S., Goh, A. Y. J., Douglas, C. P., McKelvie, P. A., Agar, A., Osher, R. H. \& Francis, I. C. (2016). Materials in the vitreous during cataract surgery: nature and incidence, with two cases of histological confirmation. CLINICAL AND EXPERIMENTAL OPHTHALMOLOGY, 44 (9), pp.797-802. https://doi.org/10.1111/ceo.12791.

Persistent Link:

http://hdl.handle.net/11343/291978 